Classification

Physics Abstracts

$06.50-07.806-42.30$

\title{
Image Analysis of Insulation Mineral Fibres
}

\author{
Hugues Talbot $\left({ }^{1}\right)$, Dominique Jeulin $\left({ }^{2}\right)$ and Daniel Hanton $\left({ }^{3}\right)$ \\ ( ${ }^{1}$ ) CSIRO Division of Mathematics and Statistics, Locked Bag 17, North Ryde, NSW 2113, Australia \\ $\left({ }^{2}\right)$ Centre de Morphologie Mathématique, ENSMP, 35 Rue Saint-Honoré, \\ 77305 Fontainebleau Cedex, France \\ $\left({ }^{3}\right)$ Centre de Recherches Industrielles de Rantigny, BP 19, 60290 Rantigny, France
}

\begin{abstract}
Résumé. - Les mesures de diamètres et les longueurs de fibres artificielles de composition vitreuse utilisées dans certains materiaux d'isolation sont importantes pour l'industrie de la laine de verre et de roche. Ces mesures sont utilisées pour des applications de contrôle de qualité. Afin d'obtenir des indicateurs de qualité fiables, la mesure de plusieurs centaines de fibres est nécessaire. Traditionellement, ces mesures sont effectuées manuellement, ce qui est une opération tout à fait fiable si l'opérateur est compétent, mais longue et répétitive. Dans cet article sont présentées deux méthodes pour réaliser ces mesures. Ces méthodes sont basées sur l'analyse automatique de micrographie électronique à balayage. Pour chacune des deux méthodes, la préparation de l'échantillon et les algorithmes d'analyse d'images utilisés pour la segmentation des fibres et leur mesure sont présentés successivement.
\end{abstract}

\begin{abstract}
The measurement of the diameters and lengths of Man-Made Vitreous Fibres (MMVFs) used in insulation materials is important to the glass and mineral wool industry. Such measurements are needed for quality control purposes. To obtain reliable quality estimators, the measurement of several hundreds of fibres is necessary, traditionally carried out by human operators. Manual measurements are reliable if performed by skilled operators, but are always slow and repetitive. In this paper, two methods for performing these measurements are presented. These methods are based on the automated image analysis of Scanning Electron Microscopy (SEM) images. For each of the two proposed methods, we present in succession the sample preparation and the image analysis algorithms used for the segmentation of the fibres and for their measurement.
\end{abstract}

\section{Introduction}

Man-Made Vitreous Fibres (MMVFs) are used in thermal and acoustic insulation materials such as glass wool. The measurement of the diameter and length of these MMVFs is useful for the insulation industry, and serves as a quality control tool and for process development. Hundreds of measurements are necessary to obtain useful information about the material, but manual measurements of the diameter and length of so many fibres is long and tedious, and unreliable in 
the case of insufficiently skilled or tired operators. This provides a motivation for automating the measurement process, which can be carried out using image analysis.

The majority of MMVFs used in the insulation industry appear as relatively regular elongated cylinders, with a diameter varying between about $0.2 \mu \mathrm{m}$ and about $20 \mu \mathrm{m}$, and a length varying from about $1 \mu \mathrm{m}$ to $1000 \mu \mathrm{m}$ or more. A significant minority of these fibres are in some way irregular: non cylindrical fibres (cone portions), fused fibres, broken fibres, etc.

Due mainly to the small diameter of the thinnest fibres, the use of electron microscopy techniques is a necessity to obtain high-quality images of such materials. Due to the extreme range of fibres lengths, it is difficult to measure both the diameter and the length of each fibre with accuracy in reasonable times. In this paper, two methods for measuring the diameter of MMVFs automatically using image analysis techniques are presented. The first method involves a crosssection sample preparation, while the second method uses a flat-bed sample preparation method. We will also indicate how to obtain some information on the length of the fibres when possible.

\section{Polished Section Method}

This methods allows to measure the diameter of MMVFs from cross-section images.

2.1 Sample Preparation. - A tuft of binder-free MMVFs is taken from the production site, parallelized by hand and then embedded in expoxy resin. The sample is cured, cut perpendicularly to its axis and polished to a mirror- like finish. The sample is then coated with carbon for observation in a scanning electron microscope (SEM) for observation in backscattered electron mode (BSE). A typical image produced by such a method is shown in Figure 1a. This sample preparation is delicate, but individual tasks can be performed in parallel for shorter preparation times. Experience shows that trained operators can typically produce 16 samples per day, on a half-time basis. As shown in Figure 1, the images obtained are of very high quality.

2.2 Image Analysis Algorithms. - This image quality makes the automated analysis of such samples reliable, although not simple, because of the large spectrum of diameters and the presence of irregular fibres (fused and broken fibres).

The analysis of these images was described by Talbot and Terol in [1] and by Talbot in even more details in [2]. We will here only briefly describe the main points of this method.

Fibre segmentation using weighted skeletons. - Fused fibres must be measured separately. In our case, image segmentation is the process that allows to logically separate two or more objects fused together. Mathematical morphology (MM) [3] is an image analysis theory and a set of techniques well suited to the segmentation of microscopy images, such as those in Figure 1. The main steps of the method, which is not fully developed here, are as follows and are illustrated in Figure 1:

- a threshold is chosen in the grey-level histogram of the image between the peak of the background and the one of the fibres (Fig. 1b),

- standard MM techniques for separating fused objects [4] do not work very well in the present case, because of the presence of fibres cut almost horizontally in the polished section, that look like very elongated ellipses; and because of the presence of small fibres touching larger ones (see Fig. 1c). Therefore, weighted skeletons [1] are used in the first segmentation pass. This involves looking at how the distance transform [5] of the fibres varies along the medial axis of the object. From which we can decide, with precision, whether or not to separate a given object in two or more particles (see Figs. 1d, e), 


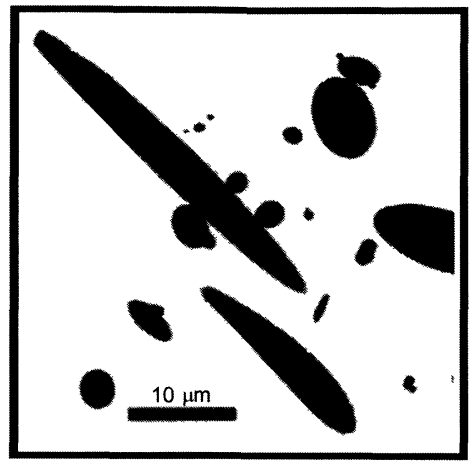

a)

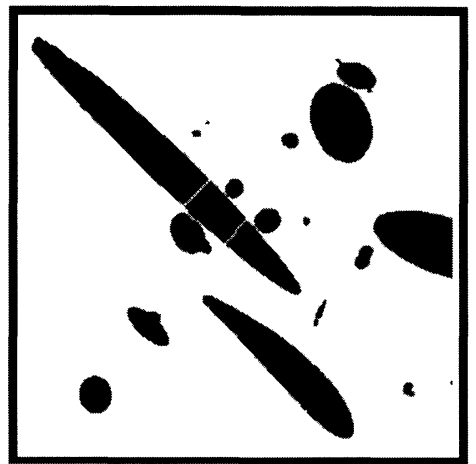

c)

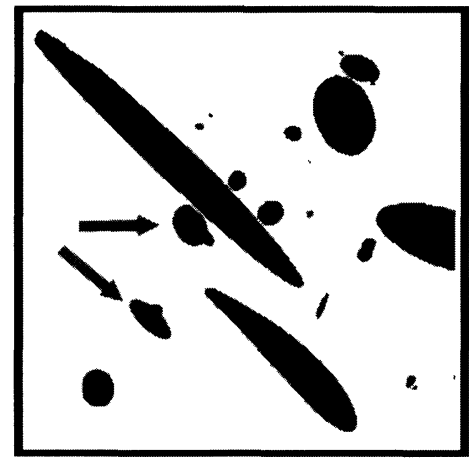

c)

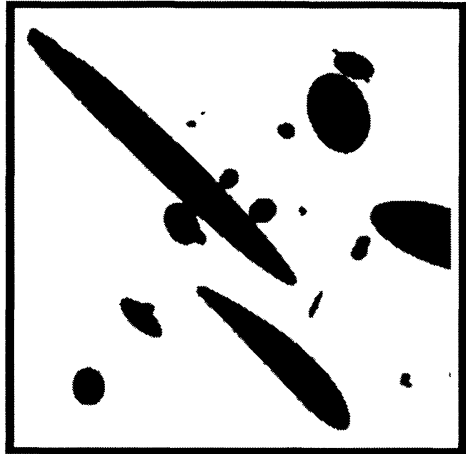

b)

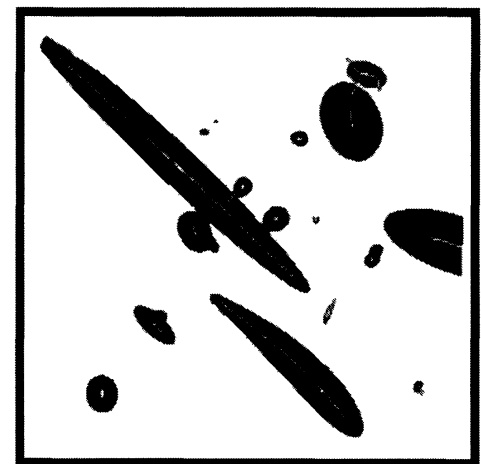

d)

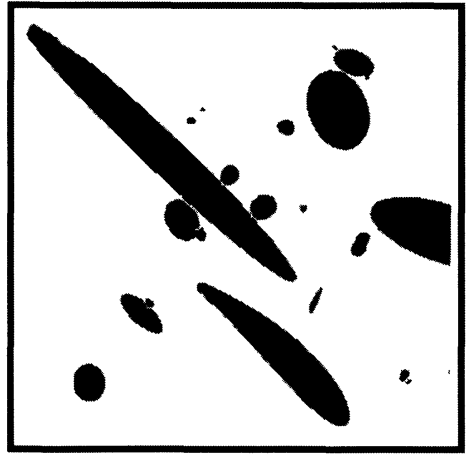

f)

Fig. 1. - Example of segmentation of MMVFs in polished section. a) original image; $b$ ) thresholded image and pre-processed image; c) tentative segmentation by filtered ultimate erosion; d) skeleton of the fibres; e) better segmentation obtained with this technique; f) best segmentation using the bisector function, which even separates the fibres shown by an arrow in (e).

- the result of this first segmentation is further enhanced by the use of the bisector function [6]. This technique is used to separate fibres that are so close together that no neck exists between them (see Figs. 1e, f). 
2.3 Measurements and Bias Correction. - The diameter of each segmented fibres is obtained by measuring the peak value of the distance transform inside each object, and doubling this value. However, this simple measurement is biased first by the usual border effect (larger fibres stand a better chance of being intersected by the field of view than thinner fibres), and second by the unknown fibre orientation distribution across diameters. The Miles-Lantuéjoul [7] method is used to correct for the first bias, and the second bias is corrected for by taking into account the orientation of each fibre, which can be obtained by measuring the ratio of the small axis of each fibre over the long axis; yielding the cosine of the fibre orientation angle.

Unbiased diameter histograms obtained with this method are quite reliable and are obtained quickly. Typically, with this method, 600 fibres can be measured per hour on a modest (PC- Pentium 90) workstation. Experimentally, reliable histograms are obtained after about 600 measurements on two different samples of the same product, i.e. 1200 measurements altogether.

\section{Flat-Bed Fibre Method}

This second method allows to get some information on the length of the fibres by measuring them as they are deposited on a filter.

3.1 Sample Preparation. - The sample preparation for this method is simpler than for the cross-section method. A tuft of binder-free MMVFs is extracted from the material. A small quantity of fibres (about $100 \mathrm{mg}$ ) is obtained with a punching tool. These fibres are dispersed in aqueous solution and filtered through a track-etched polycarbonate filter. The filter with the fibres on them is then coated for observation in a SEM in BSE mode. A typical image result is shown in Figure 2a.

3.2 Image Analysis Algorithms. - The automated image analysis for the flat-bed fibre method is more complex than for the cross-section method. It is described in details in [2]. Only the general strategy is presented here.

- Large fibres detection: large fibres are those that appear saturated white as deposited on the filter. These fibres are detected using a standard watershed algorithm [8] (see Fig. 2b). Using a high thresholding of the image as internal marker, and a low thresholding of the image (to detect the pores of the filter) as an external marker, a binary mask of where the fibres large are located is obtained,

- Parallel fibres: dark regions parallel to the edge of the large fibres in the binary mask of the large fibres are searched for. This yields parallel fibres markers, which are reconstructed according to their directional information (see Fig. 2c),

- Crossing fibres: crossing fibres are detected from the branch points of the skeleton of the binary mask. The directional information of the skeleton on either side of the crossing points is then used for reconstruction (see Fig. 2d),

- Small fibres detection: these fibres are detected using a specific linear features detector [9]. This method allows to look for linear ridge lines in grey- level images in an efficient manner (see Fig. 2e). 


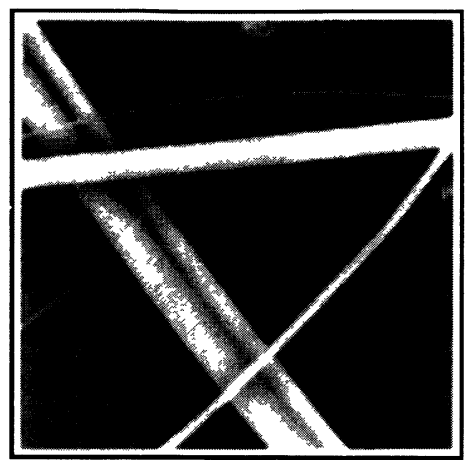

a)

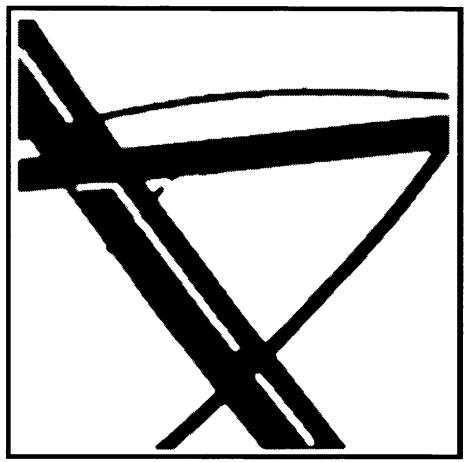

c)

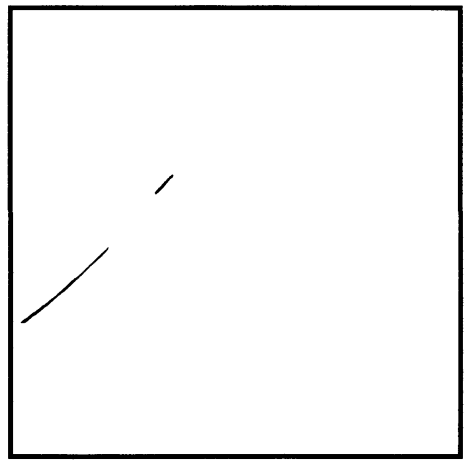

e)

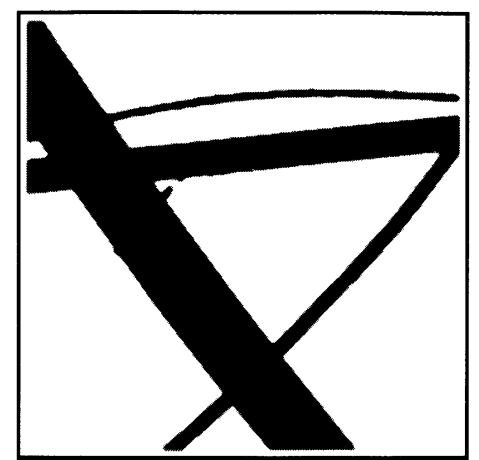

b)

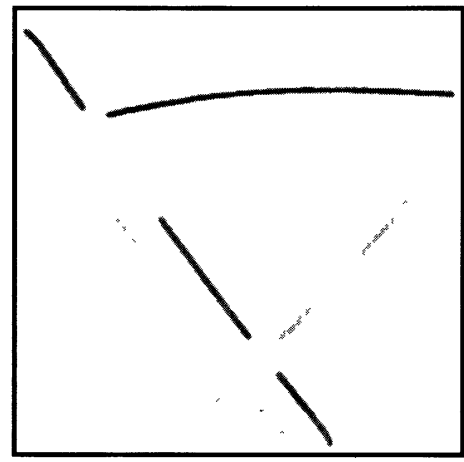

d)

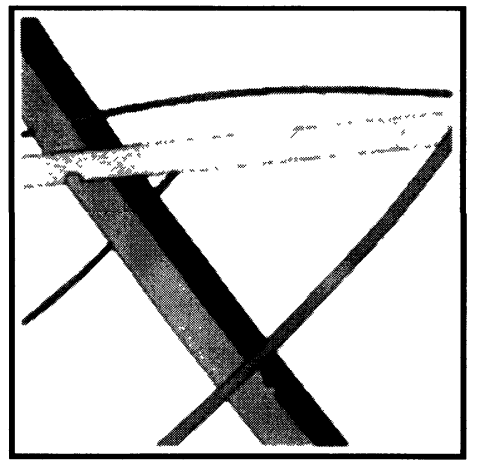

f)

Fig. 2. - Sample segmentation: a) original; b) large fibre binary mask; c) parallel fibre markers; d) reconstructed crossed fibres; e) small fibres; f) final segmentation result.

3.3 Measurements and Bias Correction. - As for the polished-section method, fibre diameter are measured using the distance transform information, averaged along the skeleton of the fibres. Fibre length is measured using the skeleton length information. In addition to the diameter and length, the number of visible fibre extremities of each fibre is also recorded. This extra information allows us to obtain an unbiased diameter histogram, as well as the mean length by diameter class. From which we build the length, area and volume weighted histograms [10].

This method enables to measure 500 fibres in two to three hours depending on the material, on a modest workstation. 


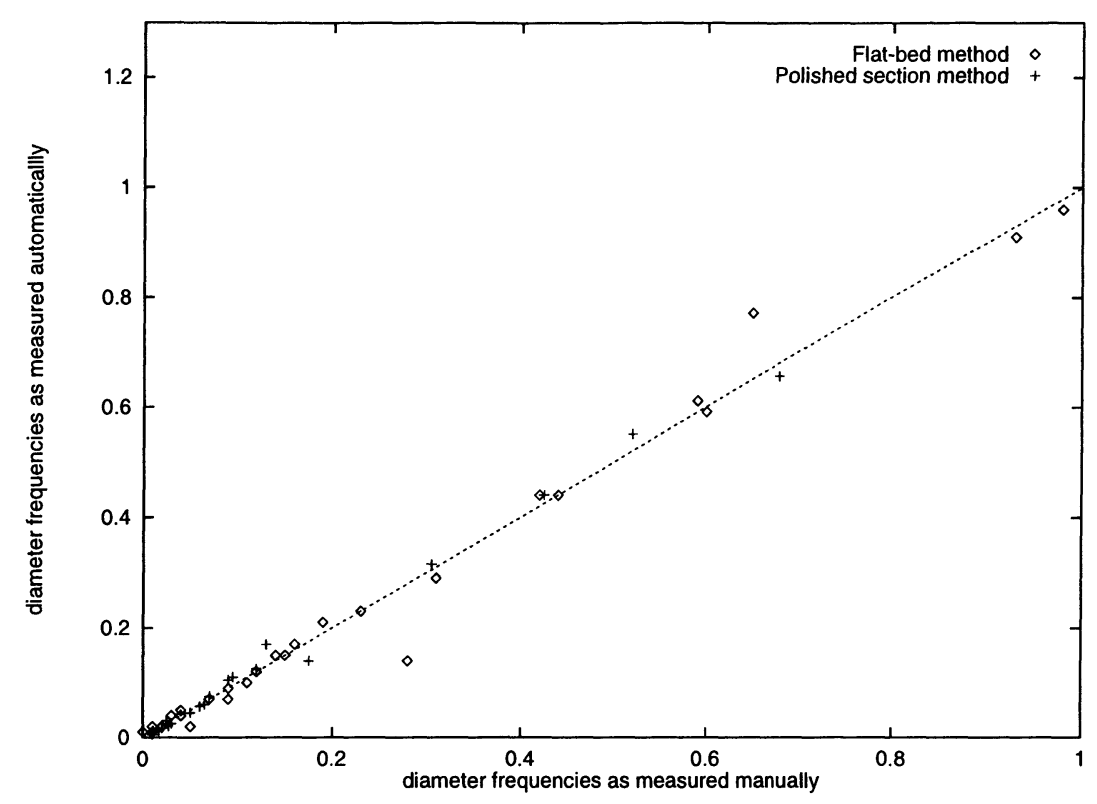

Fig. 3. - Correlation of the diameter class frequencies. Automatic versus manual.

\section{Measurement Results}

All measurements are made at a magnification of $\times 1000$ on $512 \times 512$ images. An individual pixel on these image is $0.162 \mu \mathrm{m}$ in width and height. The reference standard for both diameter and length measurements is the manual measurement. However, trying to correlate individual fibres measurements is irrelevant because human operators and automated methods do not necessarily "see" the same fibres, and when they do, both give nearly identical results. In other words, it is the segmentation step which is most important, not the measurement step.

To present meaningful comparison between human and automated measurement, we have correlated the diameter class frequencies of several histograms as given by both automated methods with the same diameter class frequencies obtained by manual measurements. Both manual and automated measurements were made from the same images of a variety of MMVF products. The frequencies of two histograms for the polished section method (corresponding to about $1600 \mathrm{fi}$ bre measurements) and of three different histograms for the flat-bed method (corresponding to about 2000 fibre measurements) were merged. The frequencies as measured automatically were correlated to the frequencies as measured manually as shown in Figure 3. As seen in this figure, the correlation is excellent, except for two points obtained with the flat-bed method. These correspond to the first two diameter class of a product with extremely fine fibres, for which measurements classes were $0.5 \mu \mathrm{m}$ wide. When both classes are merged to a 1-micron width, both manual and automated measurements agree again. Both human operators and automated methods have problems measuring accurately such small diameters.

Figure 4 shows an identical correlation drawn for the length measurements obtained with the flat-bed method on about 300 measurements. Measurements are integrated over diameter classes for the same reason as above. Only the visible length measurements of each fibre is used, as this is all that human operators can measure. In this figure, the correlation is not quite as good as for diameter measurements, but still acceptable. Also automated measurement appear systematically 


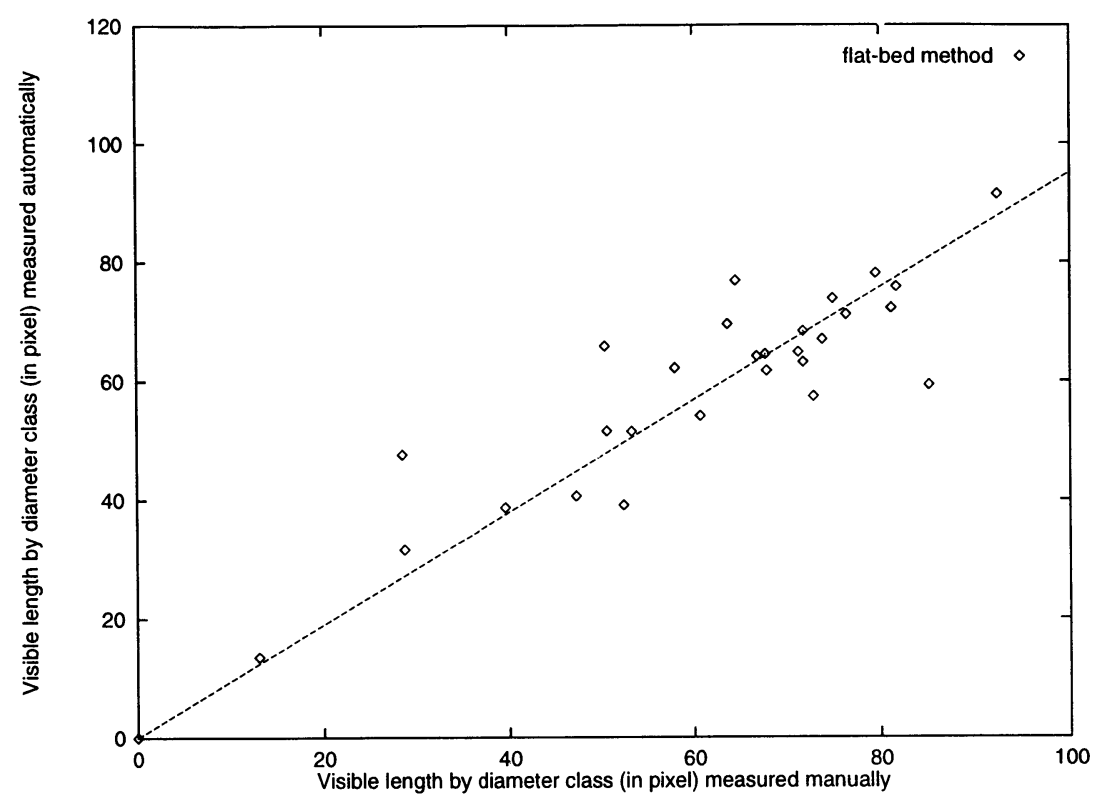

Fig. 4. - Correlation of the length measurement. Automatic versus manual.

shorter by about $5 \%$. This has little influence as this difference is the same across all classes, and for the purpose of histogram weighting, only relative measurements are necessary.

\section{Conclusion}

We have presented two methods for the automated measurements of MMVFs. The first method allows diameter measurements in cross-sections, with a complex sample preparation but an easy and rapid image analysis technique. It yields only length-weighted histograms, but the individual tasks in the sample preparation can be performed in parallel for shorter preparation times.

The second method allows both diameter and length measurements on flat-bed fibres. The sample preparation is easy and rapid, but the segmentation and measurements steps are complex, and hence slower than with the first method. However, this method can yield a variety of histograms: unweighted, length, area or volume weighted in addition to some length information.

Both method yield results which are well correlated with careful human measurements, and hence appear reliable. We believe that both methods are actually complementary. The first method could be used in day-to-day process control procedures, while the second method, slower but which yields more information on MMVF products, could be used to confirm particular results obtained with the first method, possibly in a process development environment.

\section{References}

[1] Talbot H. and Terol Villalobos I., in "Image algebra and morphological image processing III", SPIE 1769 (San Diego, CA, 1992).

[2] Talbot H., Analyse morphologique de fibres minérales d'isolation, Thèse de Doctorat de l'École des Mines de Paris (Octobre 1993). 
[3] Serra J., Image Analysis and Mathematical Morphology (Academic Press, 1982).

[4] Beucher S. and Vincent L., Traitement d'image en microscopie à balayage et en microanalyse par sonde électronique, edited by ANRT (Groupement Microanalyse et MEB), Paris (March 1990) pp. F1-F43.

[5] Rosenfeld A. and Pfaltz J.L., Patt. Recogn. 12 (1968) 33.

[6] Talbot H. and Vincent L., in "Visual Communications and Image Processing'92", SPIE 1818 (Boston, 1992) 862.

[7] Miles R., in "Stochastic Geometry" E.F. Harding, D.G. Kendall, Eds. (J. Wiley and sons, New-York, 1974).

[8] Meyer F. and Beucher S., J. Visual Comm. Image Represent. 1 (1990) 21.

[9] Talbot H., in "Mathematical Morphology and its Applications to Image and Signal Processing" (Kluwer Acad. Boston, 1996).

[10] Talbot H. and Jeulin D., Acta Stereol. 13-2 (1993) 357. 